\title{
Depressão: uma possível consequência adversa do trauma crânio-encefálico para o cuidador familiar*
}

\author{
Depression: a possible adverse consequence of the \\ traumatic brain injury for the family caregiver \\ Depresión: una posible consecuencia adversa del
trauma cráneo-encefálico para el cuidador familiar
}

Edilene Curvelo Hora Serna ${ }^{1}$, Regina Márcia Cardoso de Sousa ${ }^{2}$

\begin{abstract}
RESUMO: Estudo descritivo com abordagem quantitativa, desenvolvido no ambulatório do Trauma de Crânio com 50 cuidadores e 50 vítimas de Trauma Crânio-Encefálico, com o objetivo de verificar entre os cuidadores familiares a presença de sintomas depressivos e sua associação com o tempo decorrido do evento traumático e a condição da vítima seis meses ou mais após o trauma. O Inventário de Depressão de Beck foi aplicado aos cuidadores e a Escala de Resultados de Glasgow na avaliação da condição das vítimas, aplicados o teste Qui-quadrado de homogeneidade e o coeficiente de correlação de Pearson. Dos cuidadores avaliados 34\% apresentaram resultados sugestivos de depressão, não houve associação entre a categorização pelo Inventário de Beck e classificação na Escala de Resultados de Glasgow e tempo do trauma, ou seja, a presença de sintomas depressivos no cuidador não parece estar relacionada com o estado da vítima e o tempo decorrido após o evento traumático.
\end{abstract}

Descritores: Traumatismos cerebrais; Depressão; Cuidadores; Enfermagem familiar

ABSTRACT: Descriptive study, with quantitative approach, developed in the Clinic of the Cranium Trauma with 50 caregivers and 50 victims of Traumatic Brain Injury, with the objective to verify the presence of depressive symptoms and its association with the passed time of the traumatic event and the condition of the victim six months or more time after the trauma. The Inventory of Depression by Beck was applied to the caregivers and Glasgow Results Scale in the evaluation of the condition of the victims, applied the test homogeneity Qui-square and the coefficient of correlation by Pearson. Of the appraised caregivers $34 \%$ presented suggestive results of depression, there was not association among the categorization for the Inventory of Beck and the classification in the Glasgow Results Scale and time of the trauma, in other words, the presence of depressive symptoms in the caregiver does not seem to be related with the condition of the victim and the passed time after the traumatic event.

Keywords: Brain Injuries; Depression; Caregivers; Family nursing

RESUMEN: Un estudió descriptivo con abordaje cuantitativo fue desarrollado en el ambulatorio de Trauma de Cráneo con 50 cuidadores y 50 víctimas de Trauma Cráneo-Encefálico, con el objetivo de verificar entre los cuidadores familiares la presencia de síntomas depresivos y su asociación con el tiempo transcurrido del evento traumático y la condición de la víctima seis meses o más después del trauma. Fueron aplicados el Inventarió de Depresión de Beck a los cuidadores y la Escala de Resultados de Glasgow en la evaluación de la condición de las víctimas, aplicando los exámenes Qui-cuadrado de homogeneidad y el coeficiente de correlación de Pearson. De los cuidadores evaluados 34\% presentaron resultados sugestivos de depresión, no hubo asociación entre la categorización por el Inventarío de Beck, la clasificación en la Escala de Resultados de Glasgow y el tiempo de trauma, o sea, la presencia de síntomas depresivos en el cuidador no parece estar relacionada con el estado de la víctima y el tiempo transcurrido después del evento traumático.

Descriptores:Traumatismos cerebrales; Depresión; Cuidadores; Enfermería de la familia

\section{INTRODUÇÃO}

O Trauma Crânio-Encefálico (TCE) destaca-se em termos de magnitude, tanto entre mortos quanto feri- dos, como uma das lesões mais freqüentes. A avaliação de dados referentes ao ano de 1997 em vítimas de TCE residentes e internadas na rede hospitalar do Município de São Paulo mostrou haver evidências de que a morbi-

\footnotetext{
* Texto extraído da Dissertação de Mestrado apresentada na EEUSP em 2002 sob o título Conseqüências do Trauma CrânioEncefálico para o cuidador familiar.A Pesquisa contou com auxílio da FAPESP.

1 Enfermeira. Professora Assistente do Departamento de Enfermagem da UFS. Doutoranda em Enfermagem pela EEUSP. E-mail: edilenechs@yahoo.com.br

2 Enfermeira. Professora Livre Docente do Departamento de Enfermagem Médico-Cirúrgica da EEUSP.
} 
mortalidade por TCE é alta, mesmo com o aprimoramento dos recursos na assistência pré-hospitalar e hospitalar ${ }^{(1)}$.

Quando ocorre o TCE em um membro da família, uma crise desenvolve-se, os sentimentos dos demais membros podem variar de frustração a total impotência. A família também pode sentir-se culpada, responsável ou até com raiva sobre o acontecido ${ }^{(2)}$.

Poucos eventos na vida podem causar mudanças tão dramáticas nos papéis, relacionamentos e objetivos como o TCE e as famílias das vítimas sofrem tanto quanto, se não mais do que as próprias vítimas ${ }^{(2)}$.

Vários estudos internacionais têm mostrado as conseqüências negativas vivenciadas pelos cuidadores, em decorrência ao cuidado das vítimas de $\mathrm{TCE}^{(3-6)}$. Entre essas conseqüências, a depressão do cuidador tem sido evidenciada ${ }^{(6-9)}$.

Essas pesquisas também mostram que os familiares próximos ainda se sentem sobrecarregados e com graves problemas cinco anos ou mais após o TCE. Existem, entretanto, controvérsias se com o passar do tempo, ocorre diminuição ou aumento do impacto da doença em suas vidas ${ }^{(3-5)}$.

Diante dessa realidade e a inexistência de pesquisas com esta especificidade em âmbito nacional, resolveuse identificar a presença de sintomas depressivos no cuidador familiar principal, tendo em vista que ele tem a responsabilidade maior e permanente da vítima sob seu cuidado e, dessa forma, deverá também, de forma mais acentuada, retratar e sofrer as conseqüências do evento traumático.

O estudo também verificou a associação entre os resultados do Inventário de Beck indicativos de depressão no cuidador com duas variáveis consideradas relevantes na literatura internacional: tempo decorrido do trauma e a condição da vítima na época em que foi realizada a avaliação do estado depressivo.

\section{CASUÍSTICA E MÉTODO}

Este estudo caracteriza-se como descritivo e com abordagem quantitativa. Foi desenvolvido no Ambulatório de Trauma de Crânio do Hospital das Clínicas da Faculdade de Medicina da Universidade de São Paulo (HCFMUSP), após aprovação em Comissão de Ética e Pesquisa. A coleta de dados foi realizada com 50 cuidadores principais e 50 vítimas de TCE, no período de junho a dezembro de 2001, por meio de entrevistas estruturadas, individuais, e análise de prontuário das vítimas agendadas para atendimento nesse serviço.

Os critérios de inclusão para os cuidadores de vítimas de TCE foram: comparecer ao Ambulatório, em decorrência ao acompanhamento da vítima; ter idade igual ou superior a 18 anos e cuidar de vítimas de TCE com idade entre 12 e 60 anos; ser cuidador familiar principal da vítima de TCE que sofreu evento traumático há pelo menos seis meses, período que o quadro da vítima já se encontra estável, segundo pesquisas que analisaram o processo de recuperação após TCE ${ }^{(10-11)}$. A participação do cuidador e vítima foi vinculada ao consentimento verbal e escrito de ambos.

Inicialmente, foi feita uma análise de todos os prontuários das vítimas agendadas para atendimento, realizando-se dessa forma uma triagem inicial, por meio da data do trauma e idade. Os dados de caracterização existentes no prontuário foram registrados em formulário e as vítimas abordadas, para verificar a presença de acompanhante, cuidador familiar principal, maior de 18 anos.

Quando os critérios de inclusão no estudo eram atendidos, o cuidador e a vítima eram entrevistados separadamente por pesquisadores distintos. Nos casos em que as vítimas eram incapazes de fornecer informações ou dar consentimento de participação na pesquisa, a colaboração do cuidador também foi solicitada para essa finalidade.

Foram utilizados dois instrumentos para as entrevistas: o primeiro foi aplicado na coleta de dados da vítima de TCE e incluiu informações demográficas e clínicas, juntamente com a Escala de Resultados de Glasgow (ERG), que permitiu identificar o grau de incapacidade da vítima; o segundo formulário foi constituído pelo roteiro de entrevista com o cuidador e incluiu dados demográficos, além do Inventário de Depressão de Beck.

A ERG foi criada por Jennett e Bond e classifica as vítimas de TCE, segundo sua capacidade funcional. Estabelece cinco categorias: morte, estado vegetativo persistente (não obedece aos comandos simples nem pronuncia qualquer palavra), incapacidade grave (consciente, mas dependente), incapacidade moderada (independente, mas incapaz) e boa recuperação (retomam a vida normal, embora possam persistir leves deficiências físicas ou mentais) ${ }^{(11)}$.

O Inventário de Depressão de Beck ${ }^{(12)}$, que foi criado por Aaron Beck e validado no Brasil por Gorenstein e Andrade $^{(13)}$, foi usado para investigar a presença de sintomas depressivos nos cuidadores. É um instrumento de auto-avaliação composto de 21 itens, cada um deles contendo quatro afirmativas com graus de intensidade de zero a três, com pontuações totais que podem variar de 0 a 63 . Pode ser auto-aplicável, entretanto, em razão da baixa escolaridade da população estudada, as questões foram lidas em voz alta e vagarosamente, sendo a leitura acompanhada pelos cuidadores para, finalmente, proceder à escolha, que era registrada pelos pesquisadores.

A análise da associação entre a variável classificação no Inventário de Depressão de Beck e a variável categoria na ERG foi feita por intermédio do teste Qui-quadrado de homogeneidade. 
Nas análises dos resultados do Inventário de Depressão de Beck, o ponto de corte usado nesse estudo foi escore acima de 20 para caracterizar como sugestivos de depressão ${ }^{(14)}$.

O estudo da associação entre o tempo de trauma da vítima e o grau de depressão do cuidador (escore do Inventário de Depressão de Beck) foi feito pelo gráfico de dispersão dessas variáveis e pelo coeficiente de correlação de Pearson.

\section{RESULTADOS}

O estudo foi realizado com 50 cuidadores de idade média de 44,90 anos e desvio-padrão de 13,19, sendo $88 \%$ do sexo feminino, $68 \%$ solteiros, $58 \%$ católicos, $60 \%$ com ensino fundamental incompleto e $64 \%$ exerciam ocupação remunerada antes do evento traumático. A metade dos cuidadores em estudo era mãe da vítima, 22\% eram esposo (a) e os demais, irmão (ã), pai, tio (a) ou filho (a).
As vítimas de TCE também foram em número de 50, com idade média de 30,60 anos e desvio-padrão de 12,08 , sendo $90 \%$ do sexo masculino, $60 \%$ casados ou amasiados, e em $74 \%$ a causa externa foi o acidente de transporte. O tempo médio decorrido do trauma na época da entrevista foi de 4,42 anos, com desvio-padrão igual a 4,82, variando entre 6 meses e 18 anos, sendo que $32 \%$ tinham mais que 5 anos. Quanto à classificação na ERG, a maioria das vítimas, 56\%, apresentou incapacidade grave, $30 \%$ demonstraram boa recuperação e a minoria, 14\%, incapacidade moderada. Nenhuma vítima dependente, em estado vegetativo persistente, foi identificada.

A análise descritiva do escore do Inventário de Depressão de Beck está apresentada nos dados na Figura 1. O escore médio dos cuidadores foi 16,36 com desvio padrão igual a 12,74; sua distribuição mostrou-se assimétrica, com maior variabilidade nos valores mais altos e ainda há pontos que se destacaram dos demais por apresentarem valores muito altos, atingindo o escore máximo igual a 50 .

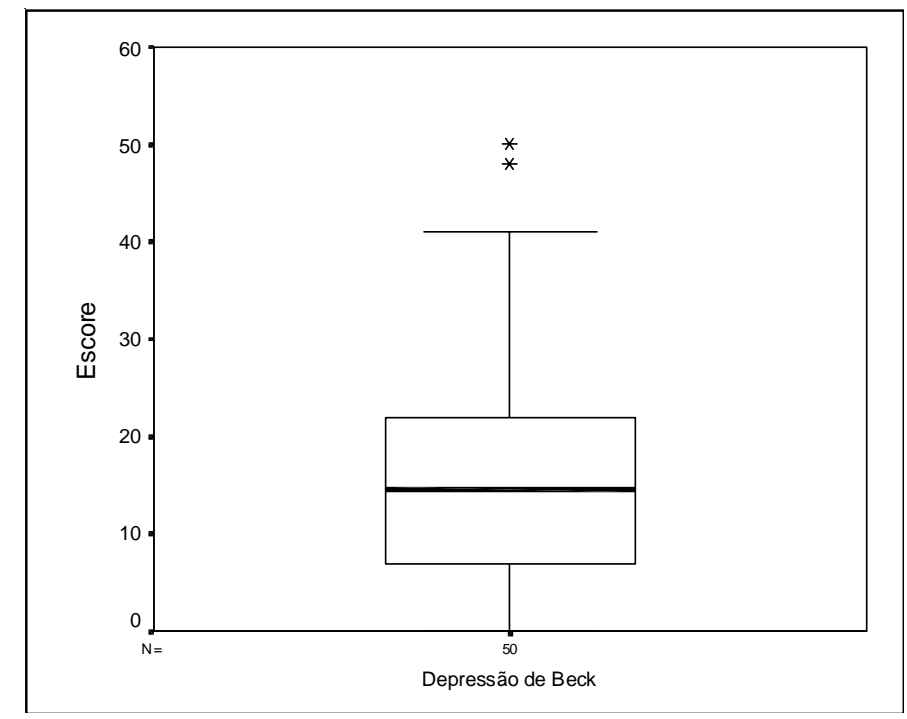

Figura 1 - Box Plots do escore do Inventário de Depressão de Beck. IC-HCFMUSP, 2001.

A categorização dos cuidadores, segundo resultado do Inventário de Depressão de Beck, revelou que 66\% dos cuidadores apresentavam pontuação menor ou igual a 20, isto é, ausência de depressão e 34\% tinham pontuação superior, indicativa de quadro depressivo.

Na Figura 2, os cuidadores de vítimas com boa recuperação, incapacidade moderada e grave segundo a ERG são distribuídos, conforme sua categorização no
Inventário de Depressão de Beck em presença ou ausência de sintomas depressivos. Observa-se que o percentual de indivíduos com indicação de quadro depressivo foi similar nos cuidadores de vítimas de TCE com boa recuperação e incapacidade moderada, 40,0 e $42,0 \%$ respectivamente, enquanto naqueles que cuidavam de indivíduos com incapacidade grave o percentual foi inferior, $28,6 \%$. 


\section{Classificação ERG (\%)}

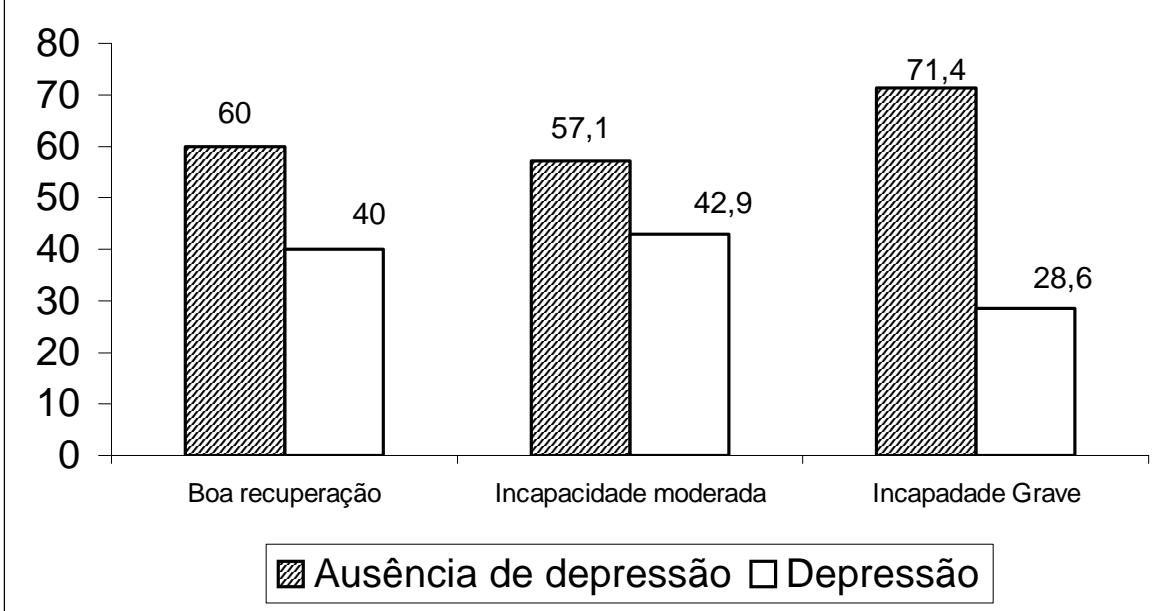

Figura 2 - Distribuição dos cuidadores, segundo as categorias do Inventário de Depressão de Beck e a classificação na Escala de Resultados de Glasgow (ERG). IC-HCFMUSP, 2001.

O teste Qui-quadrado de homogeneidade foi realizado para avaliar uma possível associação entre a categoria do Inventário de Depressão de Beck e a classificação na ERG. O teste apresentou valor de $\mathrm{p}=0,65$, indicando ausência de associação entre essas variáveis.

Para avaliar a existência de uma possível associação entre o tempo do trauma e o escore do Inventário de
Depressão de Beck, tem-se o gráfico de dispersão dessas variáveis na Figura 3, onde se observa uma nuvem de pontos sem nenhuma tendência em especial, indicando ausência de associação entre elas. Pelo coeficiente de correlação de Pearson, tem-se a confirmação de que não existe associação entre o tempo do trauma e o estado de depressão dos cuidadores $(\mathrm{p}=0,45)$.

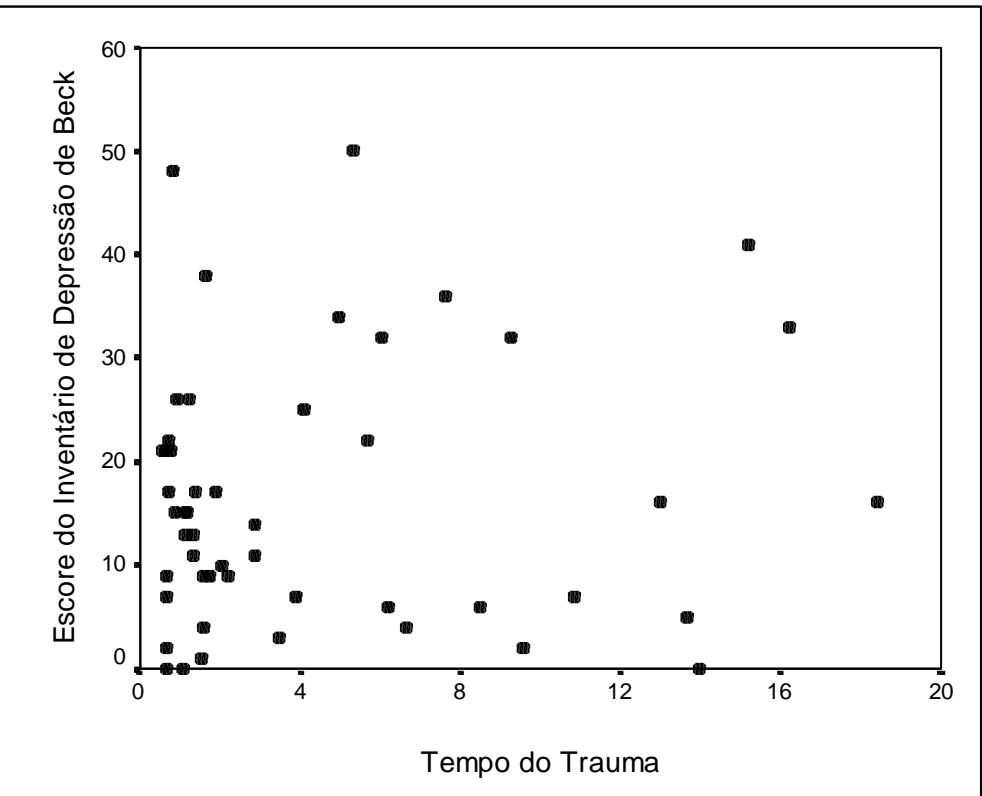

Figura 3 - Gráfico de dispersão do tempo do trauma (anos) e o escore do Inventário de Depressão de Beck. IC-HCFMUSP, 2001.

\section{DISCUSSÃO}

A presença de sintomas depressivos foi avaliada no estudo por meio do Inventário de Depressão de Beck, sendo detectada em 34\% dos cuidadores.
Resultados apresentados na literatura internacional, utilizando o Inventário de Depressão de Beck em grupos de cuidadores de vítimas de TCE, mostraram valores semelhantes de freqüência de estado depressivo: $32 \%{ }^{(8)}$, $37 \%^{(6)}$ e $37,3 \%{ }^{(9)}$; aqueles que avaliaram a presença de 
depressão por meio de outras escalas apresentaram valores um pouco menores: $27 \%^{(15)}$ e $23 \%^{(7)}$.

Pesquisadores $^{(7,16)}$ têm revelado que quanto maior o suporte social dado à família, menor o nível de depressão apresentado pelos seus membros. Afirmam também que a efetividade do suporte social é um moderador entre os escores das escalas de depressão e efeitos adversos do TCE para a família.

Vale ressaltar que esse suporte pode ser iniciado até mesmo na fase aguda, pois "a hora de ouro do trauma”, que se aplica à vítima do trauma, também se utiliza para a sua família, já que ela também necessita ser vista desde a primeira hora de chegada ${ }^{(17)}$.

O presente estudo, além de revelar em nosso meio resultado sugestivo de sintomas depressivos dos cuidadores, mostrou que não houve associação entre a categorização pelo Inventário de Beck e a classificação na ERG, como, também, com o tempo do trauma da vítima de TCE.

Resultados similares foram encontrados em estudo(7) realizado na Nova Zelândia, que analisou a associação entre depressão e inúmeras variáveis, entre elas, idade, sexo, condição sócio-econômico, tempo decorrido e gravidade do trauma, concluindo que nenhuma das características analisadas tinha relação significante com a presença da depressão no cuidador.

\section{CONCLUSÃO}

Foi observada presença de sintomas depressivos em 34\% dos cuidadores familiares principais de vítimas de trauma crânio-encefálico, independente do estado da vítima e tempo decorrido após o evento traumático.

\section{CONSIDERAÇÕES FINAIS}

Os resultados do estudo atual servem como um alerta para que os profissionais da saúde atentem para a presença de sintomas depressivos nos familiares que cuidam de vítimas de TCE, independente do estado de recuperação das mesmas, e que planejem algum tipo de suporte à família, como forma de intervenção.

Portanto, faz-se necessário o desenvolvimento de novas pesquisas, em âmbito nacional, com presença de grupo controle e com amostras diferenciadas, incluindo variáveis demográficas e socioeconômicas, que permitam análises multivariadas, objetivando a busca de fatores de risco para quadros depressivos dos cuidadores e detecção precoce de grupos com necessidade de suporte especial para o cuidado da vítima.

\section{REFERÊNCIAS}

1. Koizumi MS, Lebrão ML, Mello-Jorge MPH, Primerano V. Morbimortalidade por traumatismo crânio-encefálico no município de São Paulo. Arq Neuro Psiq 2000; 58 (1):81-9.

2. Dufor LT, Aiken LC, Gueldner S. Traumatic brain injury: a family experience. J Neurosc Nurs 1992; 24(6):317-23.

3. Brooks N, Campsie L, Symington C, Beattie A, Mckinlay W. The five year outcome of severe blunt head injury: a relative's view. J Neurol Neurosurg Psychiatry 1986; 49 (7):764-70.

4. Brzuzy S, Speziale BA. Persons with traumatic brain injuries and their families: living arrangements and well-being post injury. Soc Work Health Care 1997; 26 1):7-89.

5. Hall KM, Karzmark P, Stevens M, Englander J, O’hare P, Wright J. Family stressors in traumatic brain injury: a twoyear follow-up. Arch Phys Med Rehabil 1994; 75(8):876-84.

6. Marsh NV, Kersel DA, Havill JH, Sleigh JW. Caregiver burden at 6 months following severe traumatic brain injury. Brain Inj 1998; 12(12):1045-59.

7. Harris JK, Godfrey HP, Partridge FM, Knight RG. Caregiver depression following traumatic brain injury. Brain Inj 2001; 15(3):223-38.

8. Marsh NV, Kersel DA, Havill JH, Sleigh JW. Caregiver burden at 1 year following severe traumatic brain injury. Brain Inj 1998; 12(12):1045-59.

9. Perlesz A, Kinsella G, Crowe S. Psychological distress and family satisfaction following traumatic brain injury: injured individuals and their primary, secondary, and tertiary carers. J Head Trauma Rehabil 2000; 15(3):909-29.

10. Choi, SC, Barnes TY. Predicting outcome in the head injury patient. In: Narayan RK, editor. Neurotrauma. New York: Mac Graw Hill; 1996. cap. 53, p. 779-92.

11. Sousa RMC, Koizumi MS. Recuperação das vítimas de trauma craniencefálico entre 6 meses e 1 ano. Arq Bras Neurocir 1998; 17(2):72-80.

12. Beck AT, Rush AJ, Shaw BF, Emery G. Terapia cognitiva da depressão. Rio de Janeiro: Zahar; 1982.

13. Gorenstein C, Andrade L. Inventário de depressão de Beck: propriedades psicométricas da versão em português. Rev Psiq Clin 1998; 25(ed. esp):245-50.

14. Kendall PC, Hollon SD, Beck AT, Hammen CL, Ingram RE. Issues and recommendations regarding use of the Beck Depression Inventory. Cogn Ther Res 1987; 11(3):289-99.

15. Knight RTG, Devereux R, Godfrey H. Caring for a family member with a traumatic brain injury. Brain Inj 1998; 12(6):467-81.

16. Pelletier PM, Alfano DP. Depression, social support and family coping following traumatic brain injury. Brain Cogn 2000; 44(1):45-9.

17.Washington GT. Families in crisis. Nurs Manage 2001; 32(5):28-33. 